\title{
Diagnóstico de indicadores entéricos en cilantro (Coriandrum sativum) y perejil (Petroselinum sativum) que se expenden en mercados populares del norte de la ciudad de Quito
}

\author{
(Diagnostic of enteric indicators in coriander (Coriandrum \\ sativum) and parsley (Petroselinum sativum) sold at popular \\ food markets in Quito)
}

\author{
Dayana Cerón Salgado, Nubia Grijalva Vallejos ${ }^{1}$
}

\begin{abstract}
Resumen:
Se analizaron 108 muestras ( 54 de cilantro y 54 de perejil) obtenidas de manera aleatoria en tres puestos de expendio de tres mercados del norte de la ciudad de Quito seleccionados para este estudio. Se realizó la determinación de Coliformes Totales (CT), Coliformes Fecales (CF) y Escherichia coli (EC). La población de CT para cilantro fue de $5.09 \log$ UFC/g y $6.39 \log$ UFC/g para perejil. Se obtuvieron valores de CF de $607.0 \mathrm{NMP} / \mathrm{g}$ en el mercado uno, $596.7 \mathrm{log} \mathrm{NMP} / \mathrm{g}$ en el mercado dos y $474.2 \mathrm{NMP} / \mathrm{g}$ para el mercado tres. El $23.1 \%$ de las muestras de perejil presentaron recuentos positivos para Escherichia coli mientras que para cilantro el $14.8 \%$ de las muestras fueron positivas. La presencia de indicadores entéricos (CT, CF y EC) fue independiente del mercado y puesto de expendio lo que indica un alto nivel de contaminación y demuestra la necesidad de un control microbiológico en el sistema de riego, cosecha, transporte y condiciones higiénicas de los manipuladores para asegurar la calidad de los vegetales tipo hoja, es recomendable establecer una normativa local para evaluar la calidad microbiológica de este tipo de alimentos.
\end{abstract}

Palabras clave: Indicadores entéricos, Escherichia coli, cilantro, perejil.

\begin{abstract}
:
In this research, 108 samples were analyzed (54 of coriander and 54 of parsley). These samples were obtained under a random method in three distribution spots on each of the markets around the north of the city of Quito. Total coliforms (TC), fecal coliforms (FC) and Escherichia coli (EC) were detected. The average of TC value for coriander was $5.09 \mathrm{log} \mathrm{UFC} / \mathrm{g}$ and $6.39 \log$ UFC/g for parsley. Values of $607.0 \mathrm{NMP} / \mathrm{g}$ for $\mathrm{F}$ for the market \#1, 597.7NMP/g for the market \# 2, and $474.2 \mathrm{NMP} / \mathrm{g}$ for the market \# 3 were found. The $23.1 \%$ of the parsley samples presented positive recounts for Escherichia coli while for the coriander the $14.8 \%$ of the samples were positive for this bacterium. The presence of enteric indicators (TC, FC, and EC) was independent from the market and the distribution point which indicates a high contamination level and demonstrates the necessity of a microbiologic control on the irrigation system, farming, transport and hygienic conditions of handlers in order to assure the quality of the leafy vegetables, is recommended to set local norms to evaluate the microbiological quality of these foods.
\end{abstract}

Keywords: enteric indicators, Escherichia coli, plant leaf type, coriander, parsley.

\footnotetext{
${ }^{1}$ Universidad Tecnológica Equinoccial, Quito - Ecuador (nubia.grijalva@ute.edu.ec)
} 


\section{Introducción}

Las hortalizas son un grupo de alimentos que se cultivan en huertos o sembríos, su consumo principalmente está enfocado a la alimentación por poseer innumerables propiedades nutricionales (Rivera, Rodríguez, \& López, 2009).

El valor nutricional de las hortalizas se basa especialmente en micronutrientes como potasio, calcio, hierro; las cantidades de macronutrientes no son significativas ni presentan un alto valor calórico. Las hortalizas o vegetales tipo hoja como cilantro, perejil, apio, lechuga, acelga y espinaca son empleadas en platos tradicionales o comúnmente preparados en los hogares, tal es el caso de sopas, ensaladas, comidas rápidas (Verdú \& Carazo, 2005).

El cilantro es una planta herbácea, sus hojas presentan un aspecto muy fino con sabor y aroma característicos, es una fuente importante de vitamina $\mathrm{K}$ que interviene en factores de coagulación sanguínea. Se lo considera originario del norte de África y sur de Europa. Fue una de las primeras especies que se introdujo en América para la conservación de carnes y como hierba medicinal: sus frutos maduros se usan para condimentar. En países de Latinoamérica como México, Venezuela, Colombia y Ecuador se usan las hojas frescas enteras o picadas. Además del uso culinario, en muchas culturas (India, Grecia, China y Japón) se usa el cilantro como remedio casero. En estudios realizados en la década de los noventa se le atribuyó propiedades quelantes para atrapar metales en sangre que producen envenenamiento (Ramos, 2008).

El perejil, planta herbácea, se consume de forma cruda. Es originario del Mediterráneo oriental. Los griegos y romanos lo veneraban como una planta sagrada que acompañaba las grandes festividades (Japon, 2011).Posee vitaminas como la A, B1, B2 y C aunque no son una fuente significativa, además de minerales como hierro y fósforo; su olor característico está dado por un aceite volátil Ilamado apiol (Galeazzi, 2004).

El cilantro y el perejil son vegetales de consumo habitual que se ingieren crudos. Por su naturaleza pueden ser portadores de agentes infecciosos causantes de enfermedades transmitidas por alimentos (ETAs). Por el tipo de cultivo y la manipulación previa a la comercialización pueden presentar un alto número de enterobacterias que son la causa más importante de enfermedades gastrointestinales. La detección e identificación de este tipo de microorganismos, permite tener una idea de la calidad microbiológica que presenta el producto (Galeazzi, 2004).

Las ETA's representan un gran peligro para la salud de los seres humanos. Son producidas por la ingesta de alimentos y bebidas contaminados con microorganismos y sus toxinas, muchas personas enferman y mueren alrededor del mundo por consumir alimentos insalubres (Ramírez Merida, Alfieri, Gamboa, \& Morón de Salim, 2009). 
Pese a que no se notifica un elevado porcentaje de ETA's, estas son solo una pequeña fracción de lo que ocurre en la realidad, razón por la cual no se tienen datos de la incidencia anual de las mismas, por ejemplo, se estima que en los países industrializados se informa $<10 \%$ de la cifra real, mientras que en los países en vías de desarrollo se informa solo el $1 \%$ de las cifras reales (Fuentes \& Cepedillo, 2012).

El Sistema de Información Regional para la Vigilancia Epidemiológica de las Enfermedades Transmitidas por Alimentos (SIRVETA) de la OPS/OMS manifestó que los brotes relacionados con legumbres y hortalizas representan el 2,39\% de las enfermedades de América Latina y el Caribe (Hualpa \& Castillo, 2009).

En estudios similares realizados en vegetales frescos en Venezuela, Perú y Costa Rica se detectó la presencia de microorganismos como Salmonella, Escherichia coli, Staphylococcus aureus, mohos y levaduras que fueron hallados principalmente en lechuga, tomates, cilantro, perejil y cebollas (Rivera, Rodríguez, \& López, 2009; Ramírez Merida, Alfieri, Gamboa, \& Morón de Salim, 2009; Ginestre, Rincón, Romero, Castellano, \& Ávila, 2010).

Este estudio permitió determinar la calidad microbiológica de los dos vegetales frescos consumidos con alta frecuencia, como son cilantro y perejil, en los principales mercados del norte de la ciudad de Quito.

\section{Metodología}

\subsection{Toma de muestra}

Se seleccionaron al azar tres puestos de expendio de estos vegetales. Las muestras fueron recolectadas los días sábados en el mercado La Ofelia, y los días domingos en los mercados Santa Clara y La Carolina, en las condiciones normales de manipulación y venta al público; tanto el cilantro como perejil fueron colocados en fundas plásticas por las mismas vendedoras, las muestras se tomaron de 3 lotes diferentes, durante los meses de febrero y marzo del 2014; cada muestra en 2 tomas distintas (2 réplicas).

\subsection{Análisis microbiológicos}

Se realizaron diluciones sucesivas según la norma técnica ecuatoriana INEN 1529-2 (1999). Para la inoculación de las placas se utilizaron 7 diluciones sucesivas por cada muestra.

Para el recuento de coliformes totales se aplicó el procedimiento según Yousef \& Carlstrom (2006), en cuanto al recuento de coliformes fecales y Escherichia coli se realizaron tres tipos de colimetrías según la norma técnica ecuatoriana INEN 1529-8. 


\subsection{Análisis estadísticos}

El análisis estadístico de los datos fue realizado utilizando el software estadístico INFOSTAT versión estudiantil, se utilizó un diseño factorial $A x B$, siendo la variable $A$ los mercados y $B$ los lotes; los resultados fueron procesados mediante un análisis de varianza (ANOVA), las medias fueron comparadas con la prueba de Tukey con una significancia de 0.05 .

\section{Resultados y Discusión}

\subsection{Coliformes Totales}

Para el recuento de coliformes totales se empleó el método de la determinación del número más probable $\left(48 \mathrm{~h}\right.$ a $\left.30^{\circ} \mathrm{C}\right)$ por la técnica de dilución en tubos. Según la combinación numérica obtenida con los tubos de BGBL se detectó en todas las muestras un NMP de coliformes $>1100$ UFC/g, razón por la cual se procedió a la determinación de coliformes totales por la técnica de recuento en placa. Los valores reportados en los recuentos en placa para coliformes totales se muestran en la Tabla 1.

Tabla 1. Recuento de coliformes totales en cilantro y perejil adquiridos en diferentes mercados

\begin{tabular}{|c|c|c|}
\cline { 2 - 3 } \multicolumn{1}{c|}{} & Cilantro & Perejil \\
\hline Mercado & $5.36 \pm 0.41^{\mathrm{a}}$ & $7.45 \pm 0.22^{\mathrm{a}}$ \\
\hline 1 & $4.33 \pm 0.43^{\mathrm{b}}$ & $5.52 \pm 0.54^{\mathrm{c}}$ \\
\hline 2 & $5.57 \pm 0.24^{\mathrm{a}}$ & $6.20 \pm 0.54^{\mathrm{b}}$ \\
\hline 3 & \\
\hline
\end{tabular}

En relación a los resultados de los recuentos obtenidos en cilantro, el promedio de las medias obtenidas fue de 5.09 log UFC/g presentando diferencias de 1 log entre cada uno de los mercados, el mercado 3 fue el que presentó mayor población de coliformes totales. Ginestre et al. (2010) reportaron que el $96 \%$ de las muestras presentaron coliformes totales en muestras de cilantro comercializados en mercados populares de Maracaibo, los recuentos obtenidos estuvieron en un rango de 4 - 9 log UFC/g, resultados que fueron similares a los obtenidos en el presente estudio.

El valor promedio de los recuentos de coliformes totales en perejil fue $6.39 \mathrm{log} \mathrm{UFC} / \mathrm{g}$, siendo la diferencia entre medias superior a $0.6 \mathrm{log}$ UFC/g entre los mercados. Los mayores recuentos se obtuvieron en el mercado 1. Rincón et al. (2010) analizaron la calidad microbiológica de vegetales 
tipo hoja en dos supermercados de Maracaibo-Venezuela; reportaron la presencia de coliformes totales con valores para el cilantro de $4.19 \mathrm{log}$ UFC/g y para el perejil de $4.03 \mathrm{log}$ UFC/g, según los autores esta elevada carga microbiana indica que estos vegetales pueden representar una fuente potencial de microorganismos patógenos.

Los resultados obtenidos en relación al recuento de coliformes totales por puesto de expendio en cada mercado, para cilantro y perejil, se muestran en las tablas 2 y 3 respectivamente; se obtuvieron resultados diferentes en cada uno de ellos.

Tabla 2. Distribución de la población de coliformes totales en cilantro por puesto de expendio

\begin{tabular}{|c|c|c|c|}
\hline & \multicolumn{3}{|c|}{ Log UFC/g $^{1}$} \\
\hline Puesto & Mercado 1 & Mercado 2 & Mercado 3 \\
\hline 1 & $4.88 \pm 0.01^{\mathrm{b}}$ & $4.84 \pm 0.06^{\mathrm{a}}$ & $5.83 \pm 0.1^{\mathrm{a}}$ \\
\hline 2 & $5.72 \pm 0.19^{\mathrm{a}}$ & $3.89 \pm 0.10^{\mathrm{c}}$ & $5.42 \pm 0.26^{\mathrm{a}}$ \\
\hline 3 & $5.48 \pm 0.23^{\mathrm{ab}}$ & $4.25 \pm 0.07^{\mathrm{b}}$ & $5.46 \pm 0.08^{\mathrm{a}}$ \\
\hline
\end{tabular}

media \pm desviación estándar $(\mathrm{n}=2)$

Letras diferentes indican diferencia significativa entre mercado $(p<0.05)$

Tabla 3. Distribución de la población de coliformes totales en perejil por puesto de expendio

\begin{tabular}{|c|c|c|c|}
\hline & \multicolumn{3}{|c|}{ Log UFC/g $^{\mathbf{1}}$} \\
\hline Puesto & Mercado 1 & Mercado 2 & Mercado 3 \\
\hline 1 & $7.36 \pm 0.11^{\mathrm{a}}$ & $4.87 \pm 0.12^{\mathrm{a}}$ & $6.65 \pm 0.07^{\mathrm{a}}$ \\
\hline 2 & $7.42 \pm 0.16^{\mathrm{a}}$ & $5.67 \pm 0.08^{\mathrm{a}}$ & $6.43 \pm 0.13^{\mathrm{a}}$ \\
\hline 3 & $7.59 \pm 0.37^{\mathrm{a}}$ & $6.02 \pm 0.17^{\mathrm{b}}$ & $5.53 \pm 0.10^{\mathrm{b}}$ \\
\hline
\end{tabular}

Letras diferentes indican diferencia significativa entre mercado $(p<0.05)$

Tanto para cilantro como para perejil en el mercado 2 se encontraron los valores de coliformes totales más bajos en las muestras analizadas. Al comparar los resultados de los recuentos obtenidos en cada vegetal se pudo observar de manera evidente que la contaminación microbiana fue más elevada en las muestras de perejil; tanto el mercado 1 como el 3 presentaron elevada contaminación de coliformes totales con una diferencia de medias superior a 0.9 log UFC/g.

En el Ecuador no existen normativas que definan criterios microbiológicos para vegetales frescos que se consumen crudos, realidad que se repite en la mayoría de países de América Latina; sin embargo, en normas relacionadas de México (COFEPRIS, 1994) y Perú (DIGESA, 2008) se 
establece que los niveles de aceptabilidad para coliformes totales en verduras y hortalizas son de $10^{2}$ a $10^{4} \mathrm{UFC} / \mathrm{g}$.

\subsection{Coliformes Fecales}

En la Tabla 4 se muestran los resultados de la cuantificación de coliformes fecales obtenidos por el método de NMP $\left(45.5^{\circ} \mathrm{C}\right.$ por 48 horas $)$ referentes a los tres mercados y al tipo de vegetal analizado. Los valores más altos de coliformes fecales se encontraron en los mercados 1 y 2 , posiblemente debido a que en ambos centros de expendio los puestos de venta se localizaron en un espacio al aire libre, los puestos de venta del mercado 3 se encontraron dentro de una infraestructura cerrada diseñada para el expendio y comercialización de alimentos.

Tabla 4. NMP (Número más probable) de coliformes fecales en muestras de cilantro y perejil de los diferentes mercados

\begin{tabular}{|c|c|c|c|}
\cline { 2 - 4 } \multicolumn{1}{c|}{} & \multicolumn{3}{c|}{ NMP de Coliformes Fecales / g } \\
\cline { 2 - 4 } \multicolumn{1}{c|}{ Cilantro } & 246.9 & 350.2 & Mercado 3 \\
\hline Perejil & 360.1 & 246.5 & 243.2 \\
\hline Total & 607.0 & 596.7 & 474.2 \\
\hline
\end{tabular}

Tanto en muestras de perejil así como en cilantro la presencia de coliformes fecales fue similar, presentándose en ambos vegetales en un 29.6\% de las muestras evaluadas. Ginestre et al. (2010) reportaron porcentajes de coliformes fecales superiores al $80 \%$ en muestras de cilantro y $71 \%$ en perejil mientras que Rivera, Rodríguez, \& López (2009) encontraron un porcentaje de coliformes fecales en perejil de $36.8 \%$; los valores reportados en esta investigación son inferiores a ambos resultados. En ambas investigaciones se atribuyó esta contaminación al agua de riego procedente de ríos en los que se vierte agua residual no tratada que es propagada sobre cultivos que son vendidos en mercados locales y consumidos por la población urbana y rural de las ciudades.

Los resultados obtenidos en este estudio se interpretan como no aceptables según la norma Oficial Mexicana NOM-093-SSA1 (COFEPRIS, 1994) ya que se establece que los límites máximos permisibles para este tipo de alimentos es de $100 \mathrm{NMP} / \mathrm{g}$ de coliformes fecales 


\subsection{Escherichia coli}

De las 108 muestras de vegetales tipo hoja analizadas, 41 (37,9\%) resultaron positivas para E.coli; 25 de estas fueron de perejil y 16 de cilantro. Como se observa en la Figura 1, el perejil fue el que presentó mayor porcentaje de casos positivos de E. coli en relación al otro vegetal analizado.

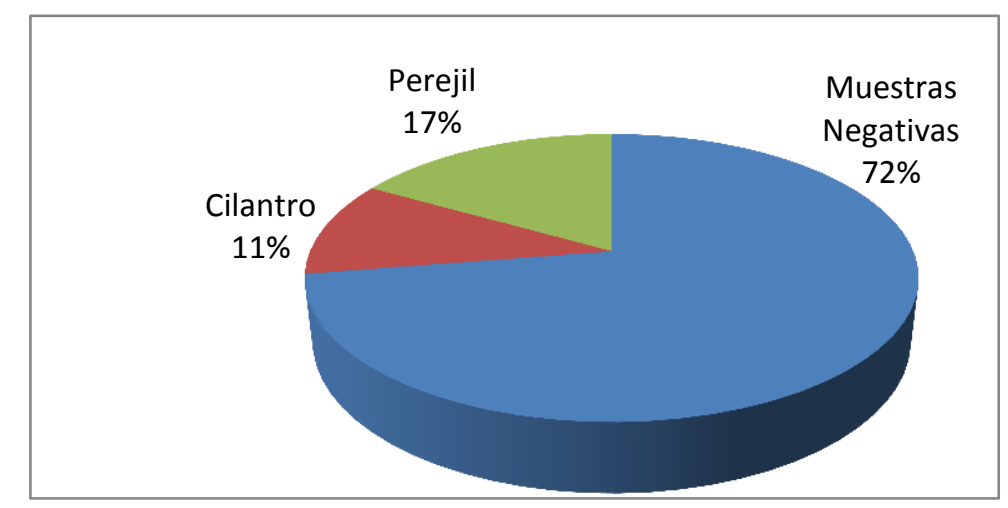

Figura 4.3. Presencia de Escherichia coli en muestras de vegetales tipo hoja analizados

Monge, Chinchilla, \& Reyes (2000) reportaron en su estudio sobre la estacionalidad de bacterias intestinales en hortalizas que se consumen crudas, que en el $42 \%$ de las muestras de hojas de cilantro, los niveles de E.coli oscilaron entre $4 \log$ UFC/g y $7 \log$ UFC/g y señalan que es necesario analizar las prácticas de manejo poscosecha ya que los índices de contaminación fecal de los productos hortícolas son mayores en las áreas de mercado que en las de cultivo.

La Norma sanitaria peruana (DIGESA, 2008) establece que para frutas y hortalizas frescas (sin previo tratamiento), el rango microbiológico aceptable para $E$. coli es de $10^{2}$ a $10^{3} \mathrm{NMP} / \mathrm{g}$; en la presente investigación se determinó que trabajando con diluciones hasta $10^{3}$ se encontró que el $37.9 \%$ de las muestras analizadas fueron positivas, criterio que determina una calidad microbiológica poco satisfactoria de los vegetales en estudio.

La presencia de la bacteria Escherichia coli en el 37.9\% de las muestras analizadas indica una defectuosa práctica higiénica de las personas que están involucradas con la poscosecha, distribución, manipulación y comercialización de estos vegetales. E.coli al ser el mejor indicador de contaminación fecal y al estar presente en alimentos puede poner en riesgo la inocuidad alimentaria.

\section{Conclusiones}

Los resultados pueden dar una idea general del nivel higiénico de los vegetales y otros productos alimenticios que se expenden en mercados y se consumen con alta frecuencia; la presencia de CT indica falencias en la aplicación de buenas prácticas de higiene durante la manipulación previa y la venta; sin embargo la presencia de este tipo de microorganismos en los alimentos no se considera como peligrosa para el consumidor. 
La presencia de coliformes fecales indica una deficiencia higiénica de las personas que manipulan los alimentos así como las condiciones insalubres de los mercados donde se comercializan vegetales frescos, las muestras positivas de $E$. coli fueron independientes del mercado y puesto de expendio por lo que se evidencia la importancia de un control microbiológico de los vegetales tipo hoja desde la cosecha para evitar brotes de enfermedades transmitidas por este tipo de alimento.

\subsection{Perspectivas futuras}

Los organismos de control tanto nacionales como internacionales deben establecer criterios microbiológicos que indiquen valores máximos permisibles de estos microorganismos en vegetales tipo hoja con la finalidad de garantizar la inocuidad para los consumidores, además de fomentar la capacitación de los agricultores y comerciantes en lo que se refiere a Buenas Prácticas Agrícolas (BPA) y Buenas Prácticas de Manufactura (BPM) durante la recolección, almacenamiento, transporte y comercialización con la finalidad de asegurar la inocuidad del producto reduciendo los excesivos niveles de contaminación. Este tipo de estudios se puede realizar en otro tipo de vegetales donde se analice la calidad microbiológica ya que estos alimentos son de consumo masivo e ingeridos crudos o cocidos, por lo que es necesario contar con rangos establecidos.

\section{Bibliografía}

Camelo, A. (2003). Manual para la preparación y venta de frutas y hortalizas. Boletín de Servicios Agrícolas de la FAO.

COFEPRIS, A. (1994). Norma Oficial Mexicana NOM-093-SSA1-1994, Bienes y Servicios, prácticas de higiene y Sanidad en la preparación de los Alimentos que ofrecen en establecimientos fijos. MEXICO DF

DIGESA. (2008). Norma que Establece los criterios microbiológicos de la calidad sanitaria eilnocuidad para los alimentos y bebidas de consumo humano. Lima-Perú.

Flores, G., \& Rojas, R. A. (2005). Enfermedades transmitidas por alimentos y PCR: prevención y diagnóstico. Salud Pública de México, 388-390.

Fuentes, J., \& Cepedillo, L. (2012). Enfermedades Transmitidas por Alimentos (ETA). Definición e Historia. La Habana- Cuba.

Galeazzi, V. O. (2004). En U. d. Alimentos, Análisis y simulación de procesos de secado de cilantro, perejil y epazote bajo condiciones de temperaturas cambiantes como requisito 
parcial para obtener el título en Licenciatura en Ingeniería en Alimentos (págs. 9-10). Cholula Puebla.

Ginestre, M., Rincón, G., Romero, S., Castellano, M., \& Ávila, Y. (2010). Calidad microbiológica y bacterias enteropatógenas en vegetales tipo hoja. Kasmera, 98-105.

Hualpa, D., \& Castillo, M. (2009). Consumo de Lácteos sin Procesar. Un riesgo latente. Zona Alimentarya, 1-4.

INEN. (1990). Control Microbiológico de los Aimentos. Determinación de Coliformes Fecales y E.coli. NTE INEN 1529-8. Quito, Ecuador.

Japon, J. (2011). Cultivo de Perejil y HierbaBuena. Hojas Divulgadoras del Ministerio de Agricultura, Pesca y Ganadería.

Ramírez Merida, L. G., Alfieri, A. Y., Gamboa, O., \& Morón de Salim, A. (2009). Frecuencia de Listeria monocytogenes en muestras de tomates y cilantro frescos en tres supermercados. Archivos Latinoamericanos de Nutrición, 318-324.

Ramos, G. S. (2008). El cilantro (Coriandum sativum) como planta medicinal emergente. inFÁRMAte, 1-5.

Rivera, M., Rodríguez, C., \& López, J. (2009). Contaminación Fecal en hortalizas que se expenden en mercados de la ciudad de Cajaramca, Perú. Perú Med, 45-48.

Verdú, J., \& Carazo, M. (2005). Hortalizas y Frutas. Mérida- Yucatán: Díaz de Santos.

Yousef, A., \& Carlstrom, C. (2006). Microbiología de los Alimentos- Manual de Laboratorio. Zaragoza: Acribia S.A. 
Enfoque UTE, V.6-N.1, Mar.2015, pp.45 - 54 